\title{
MATRICES WHOSE POWERS ARE $M$-MATRICES OR $Z$-MATRICES
}

\author{
SHMUEL FRIEDLAND, DANIEL HERSHKOWITZ, AND HANS SCHNEIDER
}

\begin{abstract}
A matrix $A$ all of whose (positive) powers are $Z$-matrices is called here a $Z M$-matrix. A matrix is called a $Z M A$-matrix if all powers of $A$ are irreducible $Z$-matrices. We prove that the spectrum of a $Z M A$-matrix is real and only the eigenvalue minimal in absolute value may be negative. By means of an operation called inflation which generalizes the Kronecker product of two matrices, we determine the class of $Z M A$-matrices of order $n$ in terms of the classes of $Z M A$-matrices of smaller orders. We use this result to show that a $Z M A$-matrix is positively diagonally similar to a symmetric matrix. Similar results hold for $M M A$-matrices which are defined in analogy with $Z M A$-matrices in terms of $M$-matrices, and for $Z M O$-matrices which are defined to be $Z M$-matrices such that all odd powers are irreducible and all even powers reducible. We also prove that a matrix is a $Z M A-, Z M O$ - or $M M A$-matrix under apparently weaker conditions. If $A$ is a real matrix such that all sufficiently large powers of $A$ are $Z$-matrices, then $A$ is a $Z M A$-matrix if $A^{2}$ is irreducible, $A$ is a $Z M O$-matrix if $A$ is irreducible and $A^{2}$ is reducible, and $A$ is an $M M A$-matrix if $A$ is an irreducible $Z$-matrix and some odd power of $A$ is an $M$-matrix.
\end{abstract}

1. Introduction. A $Z$-matrix is a real $n \times n$ matrix with all its off-diagonal elements nonpositive. An $M$-matrix may be defined as a $Z$-matrix all of whose eigenvalues have nonnegative real parts. There is a voluminous literature on $Z$ and $M$-matrices which, since such matrices are related to nonnegative matrices in an obvious manner, may be said to have originated even before the explicit definition of $M$-matrices by Ostrowski $[\mathbf{7}]$ and $Z$-matrices by Fiedler and Ptak [3]. For much information on $Z$ - and $M$-matrices see Berman and Plemmons [1].

In this paper we shall investigate matrices whose powers are $Z$ - or $M$-matrices, usually under some irreducibility condition. There is a surprisingly rich structure theory for such matrices. Before describing our principal results we shall give some definitions. By $\mathbf{R}(\mathbf{C})$ we denote the real (complex) numbers and by $\mathbf{R}^{m n}\left(\mathbf{C}^{m n}\right)$ the set of all $m \times n$ matrices with elements in $\mathbf{R}(\mathbf{C})$. From now on if we say a power of $A$ we shall mean a positive power of $A$. By $\operatorname{spec}(A)$ we shall denote the set of (distinct) eigenvalues of $A$. We say that the matrix $A$ is a direct sum of matrices $B$ and $C(A=B \oplus C)$ where other authors might say that $A$ is permutation similar to $B \oplus C$ (see the beginning of $\S 4$ ).

Received by the editors March 5, 1985 and, in revised form, April 14, 1986.

1980 Mathematics Subject Classification (1985 Revision). Primary 15A21, 15A18, 05 C50.

The research of the third author was supported in part by NSF grants MCS-80-26132 and DMS-8320189, by ONR grant N00014-85-K-1613, and by the Lady Davis Foundation of Israel while the author was a visiting professor at the Technion, Haifa, Israel. 
Definitions 1.1. Let $A \in \mathbf{R}^{n n}$.

(i) We call $A$ a $Z M$-matrix (MM-matrix) if all powers of $A$ are $Z$-matrices ( $M$-matrices).

(ii) We call $A$ a $Z M A$-matrix ( $M M A$-matrix) if $A$ is a $Z M$-matrix ( $M M$-matrix) and all powers of $A$ are irreducible.

(iii) We call $A$ a $Z M O$-matrix if $A$ is a $Z M$-matrix and all odd powers of $A$ are irreducible, while all even powers of $A$ are the direct sum of two or more matrices.

(iv) We call $A$ a $Z M E$-matrix if $A$ is either a $Z M A$-matrix or a $Z M O$-matrix.

(v) We call $A$ an eventual $Z$-matrix (eventual $M$-matrix) if for some positive $k$ the matrix $A^{r}$ is a $Z$-matrix ( $M$-matrix) whenever $r \geq k$.

(vi) We call $A$ essentially an $M M A$-matrix if there is an $s \in \mathbf{R}$ for which $A+s I$ is an $M M A$-matrix.

Our definitions of $Z M A$-, $Z M O$-, and $M M A$-matrices are motivated by results showing that $Z M$-matrices satisfying apparently much weaker irreducibility conditions belong to these classes. The strongest results of this type appear in $\S 7$ and depend on the structure theory developed in $\S 6$.

We now summarize our principal results. In $\S 2$ we investigate $Z$-matrices $A$ whose squares are also $Z$-matrices. We show that the graph of such a matrix is essentially transitive (Lemma 2.10) and it follows easily that if $A$ is also irreducible, then the off-diagonal elements of $A$ are negative (Corollary 2.14). We also characterize those patterns $S$ such that for all matrices $A$ with pattern $S$ both $A$ and $A^{2}$ are $Z$-matrices. Furthermore, in this case $A$ is a $Z M$-matrix (Theorem 2.18).

In $\S 3$ we study an eventual $Z$-matrix $A$ such that $A$ is an irreducible $Z$-matrix. Our fundamental lemma shows that $\operatorname{spec}(A)$ is real. We put

$$
\operatorname{spec}(A)=\left\{\alpha_{1}, \ldots, \alpha_{t}\right\}, \quad \alpha_{1}<\cdots<\alpha_{t},
$$

and we show that all eigenvalues are nonnegative with the possible exception of $\alpha_{1}$ which is a simple eigenvalue. Further,

$$
-\alpha_{2} \leq \alpha_{1}
$$

We then turn to the study of the class of $Z M$-matrices $A$ such that $A$ is irreducible. We show that such an $A$ is in fact a $Z M E$-matrix, and that $A$ is a $Z M A$-matrix if and only if $A^{2}$ is irreducible or the strict inequality holds in (1.3) (Corollary 3.8 and Theorem 3.6). Thus $Z M O$-matrices are characterized within the class considered by the reducibility of $A^{2}$ or by the equality in (1.3), and $M M A$-matrices by the existence of an odd power of $A$ which is an $M$-matrix or by $0 \leq \alpha_{1}$ (Theorems 3.7 and 3.9).

In $\S 4$ we introdruce an operation called inflation which generalizes the Kronecker and Hadmard products of two matrices. Suppose there is a given partition of $\{1, \ldots, n\}$ into $m$ subsets. For $C \in \mathbf{C}^{m m}$ and $U \in \mathbf{C}^{n n}$ we define a matrix $C \times \times U \in$ $\mathrm{C}^{n n}$ relative to the given partition (Definition 4.1). If $U$ belongs to a certain subset of the rank 1 partitioned matrices in $\mathbf{R}^{n n}$ (denoted here by $\mathcal{U}$; see Definition 4.3), then the operation of inflation has simple properties. Let $C \in \mathbf{C}^{n n}$ and let $U \in \mathcal{U}$ and suppose that

$$
A=(C \times \times U)+\beta G(U)=C \times \times U+\beta G(U),
$$


where $G(U)$ is a certain idempotent associated with $U \in \mathcal{U}$. Then $\operatorname{spec}(A)=$ spec $C \cup\{\beta\}$. Indeed, if the spectral decomposition of $C$ (cf. [5, Vol. I, p. 104]) is

$$
C=\left(\gamma_{1} E_{1}+Z_{1}\right)+\cdots+\left(\gamma_{t} E_{t}+Z_{t}\right)
$$

where $E_{p}$ is idempotent and $Z_{p}$ is nilpotent, $p=1, \ldots, t$, then for $U \in \mathcal{U}$ and $\beta \notin \operatorname{Spec}(C)$ the spectral decomposition of $A$ in (1.4) is given by

$$
A=\left(\gamma_{1} E_{1} \times \times U+Z_{1} \times \times U\right)+\cdots+\left(\gamma_{t} E_{t} \times \times U+Z_{t} \times \times U\right)+\beta G(U)
$$

(Lemma 4.19).

In $\S 5$ we study completely reducible idempotent $M$-matrices $G$ such that every irreducible component of $G$ is singular (called a totally singular $M$-idempotent). We show that such an idempotent is of the form $G(U)$, where $U$ is a positive element of $U$ defined above, and that $U$ is unique under an appropriate normalization (Lemma $5.5)$.

Our main results are contained in $\S 6$. If $A$ is a $Z M A$-matrix of order $n, n>$ 1 , with spectral radius $\rho(A)$, then there exist a unique partitioning of $\{1, \ldots, n\}$ into $m$ subsets, $1 \leq m<n$, a unique normalized positive $U \in \mathcal{U}$, and a unique $Z M A$-matrix $C \in \mathbf{R}^{m m}$ whose spectral radius satisfies $\rho(C)<\rho(A)$ such that $A=C \times \times U+\rho(A) G(U)$ (Theorem 6.12). Thus the class of all $Z M A$-matrices of order $n$ may be determined from the classes of $Z M A$-matrices of orders less than $n$. It follows (Theorem 6.18) that a $Z M A$-matrix may be constructed by inflation starting with a matrix

$$
C_{1}=\alpha_{1}
$$

of order 1 , successively forming

$$
C_{p}=C_{p-1} \times \times U_{p}+\alpha_{p} G\left(U_{p}\right), \quad p=2, \ldots, t,
$$

where $U_{p}$ is a matrix in $\mathcal{U}$ of appropriate dimension, and then setting

$$
A=C_{t} .
$$

The matrices $C_{p}, p=1, \ldots, t$, and normalized $U_{p}, p=1, \ldots, t$, obtained in this manner are unique. Conversely, a matrix $A$ obtained by inflation in the above manner is a $Z M A$-matrix. We then deduce that the spectral decomposition of a $Z M A$-matrix $A$ is of form

$$
A=\alpha_{1} E_{1}+\cdots+\alpha_{t} E_{t},
$$

where the $E_{p}, p=1, \ldots, t$, are themselves given by inflation in a simple manner (Corollary 6.25). As another application we deduce (Corollary 6.28) that any matrix $B$ obtained from a $Z M A$-matrix $A$ by replacing $\alpha_{p}$ by $\beta_{p}$ in $(1.10), p=1, \ldots, t$, remains a $Z M E$-matrix provided the inequalities (1.2) and (1.3) are maintained.

In $\S 7$ we show that a $Z M A$-matrix $(Z M O$-matrix) has unique $r$ th root which is a $Z M A$-matrix ( $Z M O$-matrix) provided $r$ is an odd positive integer, and that an $M M A$-matrix has a unique $r$ th root which is an $M M A$-matrix for all positive integers $r$ (Theorem 7.1). We also show that if $A$ is an eventual $Z$-matrix and some power of $A$ is irreducible, then $A$ is a $Z M E$-matrix (Theorem 7.7). Therefore $A$ is a $Z M A$-matrix if $A$ is an eventual $Z$-matrix and an even power of $A$ is an irreducible $Z$-matrix. 
In $\S 8$ we show that $e^{A}$ is an $M M A$-matrix if $A$ is essentially an $M M A$-matrix. Further, if $e^{A}$ is an $M M A$-matrix, then $A$ is essentially an $M M A$-matrix if and only if $\operatorname{spec}(A)$ is real (Theorem 8.7). We also show that the semigroup $\left\{e^{s A}: s>0\right\}$ consists entirely of $M M A$-matrices if and only if $A$ is essentially an $M M A$-matrix (Theorem 8.9).

In $\S 9$ we show that if $A$ is a symmetric $Z M A$-matrix, then all the matrices which occur in the inflation process (1.7)-(1.9) are symmetric and that every $Z M A$-matrix is positively diagonally similar to a symmetric $Z M A$-matrix (Theorems 9.2 and 9.6).

It may be remarked that most of our results on $Z M A$-matrices (and in particular, our main theorems in $\S 6$ ) have analogues for $Z M O$-matrices which are often not stated. This is a consequence of the result (Lemma 3.10) that $A$ is a $Z M E$-matrix if and only if $A+\varepsilon I$ is a $Z M A$-matrix for all positive $\varepsilon$.

We also remark that it is easy to find an example of a $Z M$-matrix none of whose powers is irreducible, e.g. Example 5.7. We have not investigated the theory of $Z M$-matrices without an irreducibility condition except for our results in $\S 2$. It is clear that many of our results will not hold in this more general context. For example, compare the obvious result that every nilpotent matrix is an eventual $Z$-matrix with the last theorem quoted above from $\S 7$.

We end this introduction with a natural problem: Characterize the ZMAmatrices and $M M A$-matrices of order $n$ without reference to $Z M A$-matrices of smaller order. In view of the results of $\S 9$ it is sufficient to characterize symmetric $Z M A$ - and $M M A$-matrices.

\section{2. $Z$-matrices whose squares are $Z$-matrices.}

DEFINITIONS 2.1. Let $A \in \mathbf{R}^{n n}$.

(i) The (directed) graph $\Gamma(A)$ of $A$ has $\{1, \ldots, n\}$ as its vertex set and $(i, j)$ is an arc of $\Gamma(A)$ if $a_{i j} \neq 0$. An arc $(i, j)$ of $\Gamma(A)$ is normally denoted by $i \rightarrow j$.

(ii) A path from $i$ to $j$ in $A$ of length $m$ is a sequence of $\operatorname{arcs}\left(i_{0}, i_{1}\right), \ldots,\left(i_{m-1}, i_{m}\right)$ in $\Gamma(A)$ with $i=i_{0}$ and $j=i_{m}$. We normally denote this path by $i_{0} \rightarrow i_{1} \rightarrow \cdots \rightarrow$ $i_{m}$.

(iii) The path $i_{0} \rightarrow i_{1} \rightarrow \cdots \rightarrow i_{m}$ is called a simple path if, for $l>k, i_{k}=i_{l}$ implies $k=0$ and $l=m$.

(iv) The path $i_{0} \rightarrow i_{1} \rightarrow \cdots \rightarrow i_{m}$ is called a circuit if it is simple and $i_{0}=i_{m}$.

Usually we refer to an arc (path) of $\Gamma(A)$ as an arc (path) in $A$. If there is a path from $i$ to $j$ in $A$, then there exists a simple path from $i$ to $j$ in $A$.

DEFinitions 2.2. Let $A \in \mathbf{R}^{n n}$.

(i) We call $A$ essentially graph transitive if, for every path $i \rightarrow j \rightarrow k$ with $i \neq k$ in $A$, it follows that $i \rightarrow k$ in $A$.

(ii) We call $A$ graph transitive if $i \rightarrow j \rightarrow k$ in $A$ implies that $i \rightarrow k$ in $A$.

(iii) We call $A$ essentially full if $i \rightarrow j$ in $A$ (viz. $a_{i j} \neq 0$ ) whenever $i \neq j$, $i, j=1, \ldots, n$.

(iv) We call $A$ full if $i \rightarrow j$ in $A$ (viz. $a_{i j} \neq 0$ ), $i, j=i, \ldots, n$.

(v) (As usual) we call $A$ irreducible if there is a path in $A$ from $i$ to $j$ for every $i, j, i, j=1, \ldots, n$.

(vi) We call $A$ completely reducible if there is a path in $A$ from $j$ to $i$ whenever there is a path in $A$ from $i$ to $j$. 
It is clear that $A$ is essentially graph transitive if and only if for every simple path $i \rightarrow j \rightarrow k$ with $i \neq k$ it follows that $i \rightarrow k$. Observe that every matrix in $\mathbf{R}^{11}$ and $\mathbf{R}^{22}$ is essentially graph transitive and that every matrix in $\mathbf{R}^{11}$ is essentially full. Note that an irreducible matrix is completely reducible, and that (as is easily proved and well known) a completely reducible matrix is a direct sum of irreducible matrices.

LEMMA 2.3. Let $A \in \mathbf{R}^{n n}$, and suppose that $A$ is essentially graph transitive and $i \neq j, 1 \leq i, j \leq n$. If there exists a simple path from $i$ to $j$ in $A$ of length $m$, then there exists a simple path from $i$ to $j$ of length $r$ for all $r, 1 \leq r \leq m$.

ProOF. Easy.

LEMMA 2.4. Let $A \in \mathbf{R}^{n n}$ be irreducible.

(i) The matrix $A$ is essentially full if and only if it is essentially graph transitive.

(ii) If $n>1$, then $A$ is full if and only if it is graph transitive.

ProOF. (i) If $A$ is essentially full, then it is evidently essentially graph transitive. To prove the converse, suppose that $A$ is essentially graph transitive. Let $i \neq j$, $1 \leq i, j \leq n$. Since $A$ is irreducible, there exists a simple path from $i$ to $j$ in $A$. Hence, by Lemma 2.3, there exists a path of length 1 from $i$ to $j$, viz. $a_{i j} \neq 0$.

(ii) The proof is similar.

Lemma 2.5. Let $A \in \mathbf{R}^{n n}$ and suppose that $A$ and $A^{2}$ are $Z$-matrices. Let $i \neq k, 1 \leq i, k \leq n$. Then

$$
a_{i k}\left(a_{i i}+a_{k k}\right) \leq 0
$$

and

$$
a_{i k}\left(a_{i i}+a_{k k}\right)<0
$$

if there exists a simple path $i \rightarrow j \rightarrow k$ in $A$.

PROOF. Note that the element of $A^{2}$ in position $(i, k)$ is given by

$$
a_{i k}^{(2)}=a_{i k}\left(a_{i i}+a_{k k}\right)+S
$$

where

$$
S=\sum_{h \neq i, h \neq k} a_{i h} a_{h k}
$$

Since every term of the last sum is nonnegative we have $S \geq 0$. But $a_{i k}^{(2)}$ is nonpositive and (2.6) now follows from (2.8).

If $i \rightarrow j \rightarrow k$, then one term of the sum in (2.9) is positive. Hence $S>0$ in (2.9), and (2.7) follows from (2.8).

Lemma 2.10. Let $A \in \mathbf{R}^{n n}$ and suppose that $A$ and $A^{2}$ are $Z$-matrices. Then

(i) $A$ is essentially graph transitive.

(ii) If there is a path from $i$ to $k$ in $A, i \neq k$, then

$$
\begin{gathered}
a_{i k}<0, \\
\left(a_{i i}+a_{k k}\right) \geq 0,
\end{gathered}
$$


and

$$
\left(a_{i i}+a_{k k}\right)=0
$$

only if $i \rightarrow k$ is the unique simple path from $i$ to $k$ in $A$.

ProOF. (i) Let $i \rightarrow j \rightarrow k$ be a simple path in $A$. Then $a_{i k} \neq 0$ by (2.7) in Lemma 2.5, and (i) is proved.

(ii) By Lemma 2.3 and part (i) either there exists a simple path $i \rightarrow j \rightarrow k$ in $A$ or $i \rightarrow k$ is the unique simple path from $i$ to $k$ in $A$. The results (2.12) and (2.13) follow from (2.6), (2.7), and the nonpositivity of $a_{i k}$.

Corollary 2.14. Let $A \in \mathbf{R}^{n n}$ and suppose that $A$ and $A^{2}$ are $Z$-matrices, where $A$ is irreducible. Then

(i) $A$ is essentially full.

(ii) If $n=2$, then $A$ has at most one negative diagonal element and (if it exists) its absolute value is less than or equal to the other diagonal element.

(iii) If $n>2$, then $A$ has at most one nonpositive diagonal element and (if it exists) its absolute value is less than any other diagonal element.

PROOF. (i) follows from Lemmas 2.10(i) and 2.4.

(ii) If $n=2$, then by irreducibility $1 \rightarrow 2$ and the assertions follow from (2.12).

(iii) If $n>2$, then by (i), there is a simple path of length 2 from $i$ to $k$ in $A$ for all $i, k, i \neq k, 1 \leq i, k \leq n$. Hence, by (2.12) and (2.13), $a_{i i}+a_{k k}>0$ and the assertions follow.

To illustrate the various possibilities which may arise in Corollary 2.4 we give three examples of irreducible $Z$-matrices whose squares are also $Z$-matrices.

$$
A=\left[\begin{array}{rr}
0 & -1 \\
-1 & 0
\end{array}\right], \quad B=\left[\begin{array}{rr}
2 & -4 \\
-2 & -1
\end{array}\right], \quad C=\left[\begin{array}{rrr}
-1 & -2 & -2 \\
-6 & 3 & -2 \\
-6 & -2 & 3
\end{array}\right]
$$

In fact, the matrices $A$ and $C$ are examples of $Z M O$-matrices and $B$ is a $Z M A$ matrix. The matrix $A$ above shows that we cannot improve "negative" to "nonpositive" in part (ii) of Corollary 2.14.

DEFINITION 2.15. (i) A pattern matrix $S$ is an $n \times n$ matrix with each entry in $\{-1,0,1\}$.

(ii) For a pattern matrix $S$ we define the subset $\Pi(S)$ of $\mathbf{R}^{n n}$ by $A \in \Pi(S)$ if $a_{i j}>0, a_{i j}=0, a_{i j}<0$ according as $s_{i j}=1, s_{i j}=0, s_{i j}=-1, i, j=1, \ldots, n$.

Definition 2.16. Let $A \in \mathbf{R}^{n n}$ and let $i_{0} \rightarrow \cdots \rightarrow i_{m}$ be a path in $A$. This path is said to be a positive (negative) path if $a_{i j}$ is positive (negative) for each arc $i \rightarrow j$ of the path.

LEMMA 2.17. Let $A \in \mathbf{R}^{n n}$ and suppose that every simple path in $A$ of length 2 is a circuit. Then every path in $A$ contains at most two distinct vertices.

PROOF. Without loss of generality, we prove the result for a path $i_{0} \rightarrow \cdots \rightarrow i_{m}$ in $A$, where $i_{k} \neq i_{k+1}, k=0, \ldots, m-1$. Then $i_{k} \rightarrow i_{k+1} \rightarrow i_{k+2}$ is a simple path and hence $i_{k+2}=i_{k}, k=0, \ldots, m-2$. Thus $i_{0}=i_{2}=\cdots$ and $i_{1}=i_{3}=\cdots$. 
THEOREM 2.18. Let $S \in \mathbf{R}^{n n}$ be a pattern matrix. Then the following are equivalent.

(i) Every positive path in $S$ of length 1 is closed and every negative path in $S$ of length 2 is closed.

(ii) For all $A \in \Pi(S)$, the matrix $A$ is a $Z M$-matrix.

(iii) For all $A \in \Pi(S)$, the matrices $A$ and $A^{2}$ are $Z$-matrices.

Proof. (i) $\Rightarrow$ (ii). From the first part of (i) it follows that every path $i \rightarrow j$ in $A$ with $i \neq j$ is negative. Hence every simple path $i \rightarrow j \rightarrow k$ in $A$ is negative and so, by the second part of (i), it is a circuit.

Let $m$ be a positive integer and let $i \neq j, 1 \leq i, j \leq n$. We observe that

$$
a_{i j}^{(m)}=\sum_{1 \leq i_{1}, \ldots, i_{m-1} \leq n} a_{i i_{1}} \cdots a_{i_{m-1} j} .
$$

Since every simple path in $A$ of length 2 is a circuit, it follows from (2.19) and Lemma 2.17 that

$$
a_{i j}^{(m)}=\sum_{\substack{0 \leq m_{1}, m_{2}, m_{3} \\ m_{1}+2 m_{2}+m_{3}+1=m}}\left(a_{i i}\right)^{m_{1}}\left(a_{i j} a_{j i}\right)^{m_{2}} a_{i j}\left(a_{j j}\right)^{m_{3}} .
$$

Suppose $a_{i j}^{(m)} \neq 0$. Then, by $(2.20), a_{i j} \neq 0$. Hence $a_{i j}<0$. Further $a_{j i} \leq 0$ and so $a_{i j} a_{j i} \geq 0$. Also $a_{i i} \geq 0$, for otherwise $i \rightarrow i \rightarrow j$ is a negative path contrary to (i). Similarly $a_{j j} \geq 0$. It now follows from (2.20) that $a_{i j}^{(m)}<0$. Thus $A^{m}$ is a $Z$-matrix.

(ii) $\Rightarrow$ (iii) is trivial.

(iii) $\Rightarrow$ (i). Since $A$ is a $Z$-matrix, clearly every positive path in $A$ of length 1 is closed. Suppose there exists a negative path $g \rightarrow h \rightarrow k$ such that $g \neq k$. Define an $n \times n$ matrix $A$ by

$$
a_{i j}= \begin{cases}-n & \text { if }(i, j)=(g, h) \text { or }(h, k), \\ s_{i j} & \text { otherwise. }\end{cases}
$$

Then $A \in \Pi(S)$. If $h \notin\{g, k\}$, then $\left|a_{g j} a_{j k}\right| \leq 1$ for $j \neq h$. Hence it follows from (2.21) that

$$
a_{g k}^{(2)}=\sum_{1 \leq j \leq n} a_{g j} a_{j k} \geq n^{2}-(n-1)=(n-1)^{2}+n>0 .
$$

If $h=g$, then, since $(g, h) \neq(h, k), a_{g g} a_{g k}=n^{2},\left|a_{g k} a_{k k}\right| \leq n$, and $\left|a_{g j} a_{j k}\right| \leq 1$ for $j \notin\{g, k\}$. Hence,

$$
a_{g k}^{(2)} \geq n^{2}-n-(n-2)=(n-1)^{2}+1>0 .
$$

Similarly if $h=k$ we again obtain (2.23). Thus in every case $a_{g k}^{(2)}>0$, which is a contradiction since $A^{2}$ is a $Z$-matrix.

REMARK 2.24. Condition (i) of Theorem 2.18 has the following matrix theoretic interpretation: After permutation similarity the pattern matrix $S$ is a direct sum of matrices of the following three types:

(a) $1 \times 1$ matrices,

(b) $2 \times 2$ matrices with nonnegative diagonal elements and negative off-diagonal elements, 
(c) matrices of the type

$$
T=\left[\begin{array}{cc}
T_{11} & T_{12} \\
0 & T_{22}
\end{array}\right],
$$

where $T_{11}$ and $T_{22}$ are nonnegative diagonal matrices, and $T_{12}$ is nonpositive.

3. $Z M A$-, $Z M O$-, and $Z M E$-matrices. Let $A \in \mathbf{R}^{n n}$ be a $Z$-matrix. Then $A$ has an eigenvalue $\mu(A)$ for which $\mu(A)<\operatorname{Re} \alpha$ for every eigenvalue $\alpha$ of $A$, $\alpha \neq \mu(A)$, and some associated eigenvector is nonnegative. We shall call $\mu(A)$ the minimal eigenvalue of $A$. If $A$ is irreducible, then $\mu(A)$ is a simple eigenvalue, viz. a simple zero of the characteristic polynomial of $A$ and its associated eigenvector may be chosen positive, e.g. [1, Theorem 4.16, p. 156].

By considering the Jordan canonical form of $A \in \mathbf{C}^{n n}$ (or the spectral decomposition (1.5)) it is easy to prove the following result: Let $\alpha^{p}$ be a simple eigenvalue of $A^{p}, p \geq 1$. Let $x$ be the eigenvector of $A^{p}$ belonging to $\alpha^{p}$ (which is unique up to a scalar multiple). Then $\alpha$ is a simple eigenvalue of $A$ with eigenvector $x$.

LemmA 3.1. Let $A \in \mathbf{R}^{n n}$ be an eventual $Z$-matrix and suppose that some power of $A$ is an irreducible $Z$-matrix. Then

(i) All eigenvalues of $A$ are real.

(iii) If $\operatorname{spec}(A)$ is given by (1.2), then $\alpha_{1}$ is a simple eigenvalue of $A$, its associated projection is positive, and

$$
\left|\alpha_{1}\right| \leq \alpha_{p}, \quad p=2, \ldots, t .
$$

(Thus $\alpha_{p}>0, p=2, \ldots, t$.)

PROOF. By assumption there is a $p, p \geq 1$, for which $A^{p}$ is an irreducible $Z$ matrix. Suppose $\alpha$ is an eigenvalue of $A$ such that $\alpha^{p}$ is the minimal eigenvalue $\mu\left(A^{p}\right)$ of $A^{p}$. Since $\alpha^{p}$ is a simple eigenvalue of $A^{p}$ and its associated eigenvector $x$ is positive, it follows from the remark preceding this lemma that $\dot{\alpha}$ is a simple eigenvalue of $A$ with associated eigenvector $x$.

Now suppose that $A^{r}$ is a $Z$-matrix for all $r \geq k$ and let $r \geq k$. Then $A^{r}$ is a $Z$-matrix and hence its minimal eigenvalue $\mu\left(A^{r}\right)$ has a nonnegative associated row eigenvector $y^{T}$. Thus

$$
\alpha^{r} y^{T} x=y^{T} A^{r} x=\mu\left(A^{r}\right) y^{T} x, \quad r \geq k,
$$

and, since $y^{T} x \neq 0$, it follows that

$$
\alpha^{r}=\mu\left(A^{r}\right), \quad r \geq k .
$$

Thus $\alpha^{r}$ is real for all positive integers $r \geq k$, and it follows that $\alpha$ is real.

Let $\beta$ be an eigenvalue of $A$. By (3.3) we have

$$
\operatorname{Re}\left(\beta^{r}\right) \geq \alpha^{r}, \quad r \geq k .
$$

Since this holds for all $r \geq k$ and $\alpha^{r}>0$ when $r$ is even, it follows that $\beta^{2}$ is nonnegative. Hence $\beta$ is real which proves (i). Thus (3.4) becomes

$$
\beta^{r} \geq \alpha^{r}, \quad r \geq k .
$$

We now consider (3.5) for an odd and an even $r$ and obtain respectively $\beta \geq \alpha$ and $\beta^{2} \geq \alpha^{2}$, which proves $\beta \geq|\alpha|$. Thus $\operatorname{spec}(A)$ satisfies (3.2) with $\alpha=\alpha_{1}$ a simple eigenvalue. 
We must still show that the projection $E$ associated with $\alpha$ is positive. We have shown that the column eigenvector $x$ corresponding to $\alpha$ is positive. Similarly the row eigenvector $u$ corresponding to $\alpha$ is positive. But $E=x u^{T} / u^{T} x$ and (ii) is proved.

When $A \in \mathbf{R}^{n n}$ is an eventual $Z$-matrix and some power of $A$ is an irreducible $Z$-matrix, we shall assume that $\operatorname{spec}(A)$ is given by (1.2). We now consider $Z M$ matrices. In $\S 7$ we shall return to eventual $Z$-matrices.

THEOREM 3.6. Let $A \in \mathbf{R}^{n n}$ be a $Z M$-matrix. Then the following are equivalent.

(i) $A$ is irreducible and $-\alpha_{2}<\alpha_{1}$.

(ii) $A$ is a ZMA-matrix.

(iii) $A^{2}$ is irreducible.

Proof. (i) $\Rightarrow\left(\right.$ ii). By Lemma 3.1(ii), $\alpha_{1}=\mu(A)$ is a simple eigenvalue of the $Z$ matrix $A$ and its associated row and column eigenvectors are positive. If $-\alpha_{2}<\alpha_{1}$, then $\mu\left(A^{r}\right)=\alpha_{1}^{r}$ is a simple eigenvalue of $A^{r}$ with positive associated eigenvectors and hence $[1$, Theorem 3.14 , p. 41$] A^{r}$ is irreducible, $r=1,2, \ldots$. This proves (ii).

(ii) $\Rightarrow$ (iii) is trivial.

(iii) $\Rightarrow$ (i). Suppose $A^{2}$ is irreducible. Then $\mu\left(A^{2}\right)=\alpha_{1}^{2}$ is a simple eigenvalue of $A^{2}$ and hence $\alpha_{2} \neq-\alpha_{1}$. We deduce (i) from (3.2).

THEOREM 3.7. Let $A \in \mathbf{R}^{n n}$ be a $Z M$-matrix. Then the following are equivalent.

(i) $A$ is irreducible and $-\alpha_{2}=\alpha_{1}$.

(ii) $A$ is a ZMO-matrix.

(iii) $A$ is irreducible and $A^{2}$ is reducible.

Proof. (i) $\Rightarrow$ (ii). If (i) holds, then $\mu\left(A^{r}\right)=\alpha_{1}^{r}$ is a simple or multiple eigenvalue of $A^{r}$ according as $r$ is odd or even. Since, by Lemma 3.1(ii), $\alpha_{1}^{r}$ has associated with it positive row and column eigenvectors, the complete reducibility of $A$ for all positives follows from $[1$, Theorem 3.14 , p. 41]. This proves (ii).

(ii) $\Rightarrow$ (iii) is trivial.

(iii) $\Rightarrow$ (i) follows immediately by (i) $\Rightarrow$ (ii) of Theorem 3.6 and Lemma 3.1.

CoROllary 3.8. Let $A \in \mathbf{R}^{n n}$ be a $Z M$-matrix. Then the following are equivalent.

(i) $A$ is irreducible.

(ii) $A$ is a $Z M E$-matrix.

(iii) All odd powers of $A$ are irreducible.

We now easily obtain the following result on $M M A$-matrices.

THEOREM 3.9. Let $A \in \mathbf{R}^{n n}$ be a $Z M$-matrix. Then the following are equivalent.

(i) $A$ is irreducible and $\alpha_{1} \geq 0$.

(ii) $A$ is an $M M A$-matrix.

(iii) $A$ is irreducible and there is an odd power of $A$ which is an $M$-matrix.

Proof. (i) $\Rightarrow$ (ii). By Theorem 3.6 the matrix $A$ is a $Z M A$-matrix since $-\alpha_{2}<$ $\alpha_{1}$. But $\mu\left(A^{r}\right)=\alpha_{1}^{r} \geq 0, r=1,2, \ldots$, and (ii) follows. 
(ii) $\Rightarrow$ (iii) is trivial.

(iii) $\Rightarrow$ (i). Since $A$ is irreducible we may write $\operatorname{spec}(A)$ in form (1.2) by Lemma 3.1. If $A^{r}$ is an $M$-matrix, then $\alpha_{1}^{r} \geq 0$. Since $r$ may be chosen odd it follows that $\alpha_{1} \geq 0$.

LEMMA 3.10. Let $A \in \mathbf{R}^{n n}$ be a $Z M$-matrix. Then $A$ is a $Z M E$-matrix if and only if $A+\varepsilon I$ is a $Z M A$-matrix for all positive $\varepsilon$.

Proof. Suppose $A$ is a $Z M E$-matrix and let $\varepsilon>0$. Let $r$ be a positive integer. Then $(A+\varepsilon I)^{r}$ is a positive linear combination of $I, A, \ldots, A^{r}$ and hence $(A+\varepsilon I)^{r}$ is a $Z$-matrix. Since $A$ is irreducible, $(A+\varepsilon I)^{r}$ is irreducible and therefore $A+\varepsilon I$ is a $Z M A$-matrix. Conversely, suppose $A+\varepsilon I$ is a $Z M A$-matrix for all $\varepsilon>0$. Since $A+\varepsilon I$ is irreducible, so is $A$, and $A^{r}=\lim _{\varepsilon \rightarrow 0}(A+\varepsilon I)^{r}$ is a $Z$-matrix, $r=1,2, \ldots$ Hence by Corollary 3.8 the matrix $A$ is a $Z M E$-matrix.

4. The operation of inflation. Our notation will not distinguish between a subset of $\{1, \ldots, n\}$ and its cardinality. An unordered partition $\left\{n_{1}, \ldots, n_{m}\right\}$ of $n$ (viz. $\{1, \ldots, n\}$ ) into $m$ subsets will be called an $m$-partition of $n$. By $\mathbf{C}\left(n_{1}, \ldots, n_{m}\right)$, we denote the set of all $n \times n$ complex matrices with the partition induced by $\left\{n_{1}, \ldots, n_{m}\right\}$, viz. $A_{i j}$ is the submatrix of $A \in \mathbf{C}^{n n}$ whose rows are indexed by $n_{i}$ and whose columns are indexed by $n_{j}$. We shall write $A_{i j} \in \mathbf{C}^{n_{i} n_{j}}$. Given an underlying partition $m$-partition of $n$ we shall also write $x_{i} \in \mathbf{C}^{n_{i}}$ for a vector whose elements are indexed by $n_{i}$. When displaying matrices it may be convenient to assume that the sets $n_{i}$ consist of consecutive integers. Similar notations will be used for $\mathbf{R}^{n n}$.

Clearly $G \in \mathbf{C}^{n n}$ is reducible if for some 2-partition of $n$ we have $G_{12}=0$, and completely reducible if, for some $m$-partition, the matrix $G$ is a direct sum of irreducible $G_{i i}$,

$$
G=G_{11} \oplus \cdots \oplus G_{m m}
$$

viz. $G_{i i}$ is irreducible and $G_{i j}=0$ if $i \neq j, i, j=1, \ldots, m$. We call $\left\{n_{1}, \ldots, n_{m}\right\}$ the partition that reduces $G$ and we call $m$ the reducibility of $G$ and write $\operatorname{red}(G)=m$. Note that a completely reducible matrix is irreducible if and only if $\operatorname{red}(G)=1$. We now define an operation which associates an $n \times n$ matrix denoted by $C \times \times U$ with an $m \times m$ matrix $C$, and an $n \times n$ matrix $U$ with a given $m$-partition.

DEFINITION 4.1. Let $\left\{n_{1}, \ldots, n_{m}\right\}$ be an $m$-partition of $n, U \in \mathbf{C}\left(n_{1}, \ldots, n_{m}\right)$, and $C \in \mathbf{C}^{m m}$. Then the inflation matrix of $C$ with respect to $U$ (relative to $\left.\left\{n_{1}, \ldots, n_{m}\right\}\right)$ is defined by

$$
C \times \times U=\left[\begin{array}{ccc}
c_{11} U_{11} & \cdots & c_{1 m} U_{1 m} \\
\vdots & & \vdots \\
c_{m 1} U_{m 1} & \cdots & c_{m m} U_{m m}
\end{array}\right]
$$

Note that the notation $C \times \times U$ does not make explicit the $m$-partitioning involved. Also note that $C \in \mathbf{R}^{m m}$ and $U \in \mathbf{R}\left(n_{1}, \ldots, n_{m}\right)$ implies that $C \times \times U \in$ $\mathbf{R}^{n n}$. Let $n=r m$, where $r$ is a positive integer, and let $n_{i}=r, i=1, \ldots, m$. If $U_{i j}=V, i, j=1, \ldots, r$, then $C \times \times U=C \times V$, the Kronecker product of $C$ and $V$. Thus our operation of inflation is a generalization of the Kronecker product. We shall use the inflation operation $C \times \times U$ is some cases when $U=I_{n}$, the $n \times n$ 
identity, and, most frequently, when $U$ belongs to a certain subset of the rank 1 matrices, which we now define.

DEFINITION 4.3. Let $\left\{n_{1}, \ldots, n_{m}\right\}$ be an $m$-partition of $n$. We define the subset $\mathcal{U}\left(n_{1}, \ldots, n_{m}\right)$ of $\mathbf{C}\left(n_{1}, \ldots, n_{m}\right)$ by $U \in \mathcal{U}\left(n_{1}, \ldots, n_{m}\right)$ if

$$
\operatorname{rank} U_{i i}=1 \text { and } U_{i i} \text { is irreducible, } \quad i=1, \ldots, m,
$$

and

$$
U_{i j} U_{j k}=U_{i k}, \quad i, j, k=1, \ldots, m .
$$

Equivalently, $U \in \mathbf{C}^{n n}$ belongs to $U\left(n_{1}, \ldots, n_{m}\right)$ if and only if there exist $u_{i}, v_{i} \in$ $\mathbf{C}^{n_{i}}$ such that $u_{i}, v_{i}$ have no zero components and

$$
\begin{gathered}
\left(v_{i}\right)^{T} u_{i}=1, \quad i=1, \ldots, m, \\
U_{i j}=u_{i}\left(v_{j}\right)^{T}, \quad i, j=1, \ldots, m .
\end{gathered}
$$

Note that $U_{i i}$ is idempotent and hence trace $U_{i i}=\operatorname{rank} U_{i i}=1$. The following properties are easily proved for $U \in \mathcal{U}\left(n_{1}, \ldots, n_{m}\right)$.

$$
\begin{gathered}
(\alpha C) \times \times U=\alpha(C \times \times U), \\
(C+D) \times \times U=(C \times \times U)+(D \times \times U), \\
(C D \times \times U)=(C \times \times U)(D \times \times U),
\end{gathered}
$$

where $C, D \in \mathbf{C}^{m m}$.

If $m=n$, then $U_{i j}=1 \in \mathbf{C}^{11}, i, j=1, \ldots, n$, and hence $C \times \times U=C$. (Usually $n>m$.) With every matrix $U \in \mathcal{U}\left(n_{1}, \ldots, n_{m}\right)$ we associate a matrix

$$
G=G(U)=I_{n}-\left(I_{m} \times \times U\right) .
$$

Thus

$$
G=G_{11} \oplus \cdots \oplus G_{m m}
$$

where

$$
G_{i i}=I-U_{i i}, \quad i=1, \ldots, m,
$$

and hence

$$
\operatorname{nullity}\left(G_{i i}\right)=1, \quad\left(G_{i i}\right)^{2}=G_{i i}, \quad i=1, \ldots, m .
$$

Conversely given $G \in \mathbf{C}^{n n}$ satisfying (4.12)-(4.14) with $U_{i i}$ an irreducible rank 1 matrix we can find $u_{i}, v_{i}, i=1, \ldots, m$, satisfying (4.6) and hence construct a $U \in \mathcal{U}\left(n_{1}, \ldots, n_{m}\right)$ to which $G$ corresponds, viz. $G=G(U)$. However, $U$ is not unique. Thus we call $U \in U\left(n_{1}, \ldots, n_{m}\right)$ normalized if in (4.6) we have

$$
\left\|u_{i}\right\|=\left\|v_{i}\right\|, \quad i=1, \ldots, m
$$

where \|\| is the Euclidean norm of appropriate dimension. We then have uniqueness:

LEMMA 4.16. Let $\left\{n_{1}, \ldots, n_{m}\right\}$ and $\left\{n_{1}^{\prime}, \ldots, n_{m^{\prime}}^{\prime}\right\}$ be $m$ - and $m^{\prime}$-partitions of $n$ respectively and let $U \in \mathcal{U}\left(n_{1}, \ldots, n_{m}\right)$ and $U^{\prime} \in \mathcal{U}\left(n_{1}^{\prime}, \ldots, n_{m^{\prime}}^{\prime}\right)$ be normalized. If $G=G(U)=G\left(U^{\prime}\right)$ is given by (4.11), then $m=m^{\prime},\left\{n_{1}, \ldots, n_{m}\right\}=\left\{n_{1}^{\prime}, \ldots, n_{m}^{\prime}\right\}$, and $U=U^{\prime}$.

Proof. Let $G_{i i}$ be given by (4.13), $i=1, \ldots, m$. By (4.12), since $G_{i i}$ is irreducible, $G_{i i}=I-U_{i i}^{\prime}$, after renumbering the sets of one of the partitions, 
$i=1, \ldots, m$. Thus we have $m=m^{\prime}$ and the identity of the two partitions. By (4.15), $U_{i i}$ determines $u_{i}, v_{i}$ uniquely and hence $U=U^{\prime}$.

In the rest of this section we assume that $\left\{n_{1}, \ldots, n_{m}\right\}$ is an $m$-partition of $n$.

Lemma 4.17. Let $C \in \mathbf{C}^{m m}$ and let $U \in \mathcal{U}\left(n_{1}, \ldots, n_{m}\right)$. Then

$$
\operatorname{rank}(C \times \times U)=\operatorname{rank} C .
$$

PROOF. For $x \in \mathbf{C}^{m}$ and $x^{T}=\left(x_{1}, \ldots, x_{m}\right)$ define $\varphi(x) \in \mathbf{C}^{n}$ by

$$
\varphi(x)^{T}=\left(x_{1} u_{1}^{T}, \ldots, x_{m} u_{m}^{T}\right),
$$

where $U_{i j}=u_{i} v_{j}^{T}, i, j=1, \ldots, m$. It is easy to check that $\varphi$ is a linear map of $\mathbf{C}^{m}$ into $\mathbf{C}^{n}$ which is $1-1$ since $\varphi(x)=0$ implies that $x=0$. We claim that $\varphi($ Range $C)=\operatorname{Range}(C \times \times U)$. Suppose first that $z \in \operatorname{Range} C$, say $z=C x$, where $x \in \mathbf{C}^{m}$. Direct computation shows that $(C \times \times U) \varphi(x)=\varphi(C x)=\varphi(z)$. Hence $\varphi($ Range $C) \subseteq \operatorname{Range}(C \times \times U)$. Next let $y \in \operatorname{Range}(C \times \times U)$, say $y=$ $(C \times \times U) w$, where $w \in \mathbf{C}^{n}$. Partition $w$ conformably with $U$ and define $\hat{w} \in \mathbf{C}^{m}$ by $\hat{w}_{i}=v_{i}^{T} w_{i}, i=1, \ldots, m$. Then $y=(C \times \times U) w=\varphi(C \hat{w})$. This shows that Range $(C \times \times U) \subseteq \varphi($ Range $U)$, and our claim is proved. We now deduce the lemma from the result that $\varphi$ is an isomorphism of $\operatorname{Range} C$ onto $\operatorname{Range}(C \times \times U)$.

For $C \in \mathbf{C}^{m m}$ write the spectral decomposition as

$$
C=\sum_{p=1}^{t}\left(\gamma_{p} E_{p}+Z_{p}\right)
$$

(cf. Gantmacher [5, Vol. I, p. 104]). The spectral radius of $C$ will be denoted by $\rho(C)$, viz. $\rho(C)=\max \{\gamma \mid: \gamma \in \operatorname{spec}(C)\}$.

LEMMA 4.19. Let the spectral decomposition of $C \in \mathbf{C}^{m m}$ be given by (4.18) and let $U \in \mathcal{U}\left(n_{1}, \ldots, n_{m}\right)$. Suppose that

$$
A=\beta G(U)+C \times \times U .
$$

Then

$$
A=\sum_{p=1}^{t}\left(\gamma_{p}\left(E_{p} \times \times U\right)+\left(Z_{p} \times \times U\right)\right)+\beta G(U) .
$$

Further, if $n>m$ and $\beta \notin \operatorname{Spec}(C)$, then the spectral decomposition of $A$ is given by (4.21).

ProOF. We use (4.8)-(4.10) to establish (4.21) and to verify that $G(U), E_{p} \times \times U$, $Z_{p} \times \times U$ satisfy the conditions of a spectral decomposition in the case that $n>m$ and $\beta \notin \operatorname{Spec}(C)$. Then $n_{i}>1$ for some $i, 1 \leq i \leq m$, and we have $I_{n}-G(U)=I_{m} \times \times U \neq I$. Thus $G(U) \neq 0$.

Corollary 4.22. Suppose $A$ is given by (4.20). Then $\operatorname{Spec}(A)=\operatorname{Spec}(C) \cup$ $\{\beta\}$ and the multiplicity of each eigenvalue $\alpha$ of $A, \alpha \neq \beta$, equals the multiplicity of $\alpha$ in $C_{j}$, while the multiplicity of $\beta$ as an eigenvalue of $A$ equals $n-m$ plus the multiplicity of $\beta$ as an eigenvalue of $C$.

ProOF. Use Lemmas 4.17 and 4.19.

REMARK. If $\beta \in \operatorname{Spec}(C)$ (in which case $\operatorname{Spec}(A)=\operatorname{Spec}(C)$ ), then the spectral decomposition of $A$ is obtained by adding $G(U)$ to one of the $E_{p} \times \times U, 1 \leq p \leq t$. If $m=n, G(U)=0$ and $A=C$. 
LEMMA 4.23. Let $U \in \mathcal{U}\left(n_{1}, \ldots, n_{m}\right)$ and let $G=G(U)$. Let $B \in \mathbf{C}^{n n}$. Then $B G=0=G B$ if and only if $B=C \times \times U$ for some $C \in \mathbf{C}^{m m}$.

Proof. We let $U_{i j}=u_{i} v_{j}^{T}, i, j=1, \ldots, m$, and we partition $B$ conformably with $U$. Then $B G=0=G B$ is equivalent to $B_{i j} G_{j j}=0$ and $G_{i i} B_{i j}=0$, $i, j=1, \ldots, m$, and hence, since $\operatorname{nullity}\left(G_{\imath i}\right)=1, i=1, \ldots, m$, also equivalent to $B_{i j}=c_{i j} u_{i} v_{j}^{T}=c_{i j} U_{i j}$.

5. The structure of completely reducible $M$-idempotents. In view of their importance to our results, we now describe the structure of completely reducible idempotent $M$-matrices. An idempotent $M$-matrix will be called an $M$ idempotent. We call an $M$-idempotent totally singular if it is completely reducible and each irreducible component is singular. A matrix $A$ is nonnegative $(A \geq 0)$ if all its entries are nonnegative and positive $(A>0)$ if all its entries are positive.

LEMMA 5.1. Let $G$ ben an $n \times n$ nonsingular irreducible $M$-idempotent. Then $n=1$ and $G=[1]$.

Proof. A nonsingular idempotent is the identity which is irreducible only if it is $1 \times 1$.

LEMMA 5.2. Let $G \in \mathbf{R}^{n n}$. Then $G$ is a singular irreducible $M$-idempotent if and only if

$$
G=I-U
$$

and

$$
U>0, \quad U^{2}=U, \quad \operatorname{rank} U=1 .
$$

Proof. If $G$ is of the given form, then $G$ is an irreducible $M$-matrix and $G^{2}=G$, since $U^{2}=U$. Conversely, let $G$ be an irreducible singular $M$-matrix. Then $\operatorname{rank} G=n-1$ and hence, since $G$ is idempotent, $G=I-u v^{T}$ where $G u=0$ and $v^{T} G=0$. But since $G$ is an irreducible $M$-matrix, $u>0$ and $v>0$. Hence $U=u v^{T}>0$.

In particular, if $G$ is a $1 \times 1$ singular $M$-idempotent, then $G=0$.

LEMMA 5.5. Let $G \in \mathbf{R}^{n n}$. If $G$ is a totally singular $M$-idempotent with $\operatorname{red}(G)=m$, then there is a unique m-partition $\left\{n_{1}, \ldots, n_{m}\right\}$ of $n$ and a unique normalized positive $U \in U\left(n_{1}, \ldots, n_{m}\right)$ such that $G=G(U)$, viz. $G$ and $U$ satisfy (4.11)-(4.14). The converse also holds.

ProOF. Let $\left\{n_{1}, \ldots, n_{m}\right\}$ be the $m$-partition of $n$ which reduces $G$. It is immediate by Lemma 5.2 that we may choose positive $U_{i i}, i=1, \ldots, m$, to satisfy (4.11)-(4.13). We then express each $U_{i i}$ as $u_{i}\left(v_{i}\right)^{T}$ so that (4.14) holds. Then $G=G(U)$ and $U$ is a normalized positive element of $U\left(n_{1}, \ldots, n_{m}\right)$. The uniqueness of the partition and of $U$ follows from Lemma 4.16.

To prove the converse, let $\left\{n_{1}, \ldots, n_{m}\right\}$ be an $m$-partition of $n$ and let $U$ be a normalized positive matrix in $U\left(n_{1}, \ldots, n_{m}\right)$. Then it is clear that $G=G(U)$ is an $M$-matrix and the idempotence and the total singularity of $G$ follow from (4.11)-(4.13). 
COROLLARY 5.6. If $G$ is a totally singular $M$-idempotent, then the nullity of $G$ is $\operatorname{red}(G)$.

Proof. Let $\operatorname{red}(G)=m$. By Lemma 5.5, $G$ is the direct sum of $m$ irreducible singular $M$-matrices and the nullity of each of these is 1 .

EXAMPLE 5.7. An example of an $M$-idempotent which is not completely reducible is

$$
G=\left[\begin{array}{rr}
0 & 0 \\
-1 & 1
\end{array}\right]
$$

6. The structure of $Z M A$-matrices. We now come to our main results on the structure of $Z M A$-matrices. In view of Lemma 3.10 similar results then follow for $Z M O$-matrices.

Let $A \in \mathbf{R}^{n n}$ be a $Z M A$-matrix. By Lemma 3.1 and Theorem 3.6 we shall write the distinct eigenvalues of $A$ as

$$
\alpha_{1}<\alpha_{2}<\cdots<\alpha_{t}
$$

where

$$
-\alpha_{2}<\alpha_{1}<\alpha_{2},
$$

and the spectral decomposition of $A$ as

$$
A=\sum_{p=1}^{t}\left(\alpha_{p} E_{p}+Z_{p}\right)
$$

LEMMA 6.4. Let $A$ be a $Z M A$-matrix.

(i) If $n=1$, then $t=1$ in (6.1).

(ii) If $n>1$, then $t>1$ in (6.1) and $\rho(A)>0$.

ProOf. Assertion (i) is trivial and (ii) follows from the result that the minimal eigenvalue $\alpha_{1}$ of an irreducible $Z$-matrix is simple.

We now exploit three basic results to obtain information about the spectral decomposition (6.3). First, if $r$ is sufficiently large, then

$$
A^{r}=\sum_{p=1}^{t}\left(\alpha_{p}^{r} E_{p}+\left(\begin{array}{l}
r \\
1
\end{array}\right) \alpha_{p}^{r-1} Z_{p}+\cdots+\left(\begin{array}{c}
r \\
\nu(p)-1
\end{array}\right) \alpha_{p}^{r-\nu(p)+1} Z_{p}^{\nu(p)-1}\right),
$$

where $\nu(p)$ is the index of the eigenvalue $\alpha_{p}$ of $A$, i.e. the dimension of the largest Jordan block associated with $\alpha_{p}$. It follows that since $\rho=\rho(A)=\alpha_{t}>\left|\alpha_{p}\right|$, $p=1, \ldots, t-1$,

$$
\lim _{r \rightarrow \infty}\left(A^{r} / \rho^{r} r^{\nu(t)-1}\right)=Z_{t}^{\nu(t)-1}
$$

(as is well known) and we use the convention that $E_{t}$ replaces $\left(Z_{t}\right)^{0}$ if $\nu(t)=1$ (or equivalently $Z_{t}=0$ ). Second, we note that $E_{1}$ is a positive (rank 1) matrix, since $A$ is an irreducible $Z$-matrix. Third, it follows from the usual Frobenius normal form of a $Z$-matrix that a nilpotent $Z$-matrix is nonpositive. 
LEMma 6.7. Let $A \in \mathbf{R}^{n n}$ be a $Z M A$-matrix. Then the spectral radius of $A$ is simple geometrically, viz. $Z_{t}=0$ in (6.3).

PROOF. If $n=1$ the result is trivial. Let $n>1$. Then by Lemma 6.4, $\rho(A)=\rho>0$. Suppose to the contrary that the index of $\rho$ is a positive integer $\nu>1$. Then, since $A$ is $Z M A$-matrix it follows from (6.6) that $Z=Z_{t}^{\nu-1}$ is a nonzero nilpotent $Z$-matrix and so $Z \leq 0$. But $E_{1} Z=0$ and since $E_{1}>0$ we now obtain that $Z=0$. But this is a contradiction.

We shall require the next lemma which is independent of preceding results.

Lemma 6.8. Let $A \in \mathbf{R}^{n n}$ be a $Z M$-matrix. Then

$$
\left|a_{i i}\right| \leq \rho(A), \quad i=1, \ldots, n .
$$

Proof. If $A$ is nilpotent, then, as is well known, $A$ is a permutation similar to a strictly upper triangular matrix. Hence (6.9) holds in this case.

So suppose that $A$ is nonnilpotent. Assume that (6.9) does not hold, say $\left|a_{h h}\right|>$ $\rho(A)$, where $1 \leq h \leq n$. Let $B=A /\left|a_{h h}\right|$. Then $\rho(B)<1$. Hence $\lim _{r \rightarrow \infty} B^{r}=0$. On the other hand, since $B$ is a $Z M$-matrix we have

$$
b_{h h}^{(2)} \geq\left(b_{h h}\right)^{2} \geq 1
$$

and so by induction

$$
b_{h h}^{(r)} \geq\left(b_{h h}\right)^{r} \geq 1, \quad r=2^{k}, \quad k=1,2, \ldots
$$

But this is a contradiction.

An example of an irreducible $M$-matrix $H$ (with positive spectrum) for which $\max h_{i i}>\rho(H)$ is given by

$$
\dot{H}=\left[\begin{array}{rrr}
5 & -1 & 0 \\
0 & 2 & -1 \\
-1 & -1 & 2
\end{array}\right]
$$

Here $\operatorname{spec}(H)=\{.885,3.254,4.861\}$ to an accuracy of 3 places of decimals. It is easily seen that $H^{2}$ is not a $Z$-matrix.

LEMMA 6.10. Let $A \in \mathbf{R}^{n n}$ be a $Z M A$-matrix.

(i) If $n=1$, then the projection $E_{t}$ associated with $\rho(A)$ is $1 \in R^{11}$.

(ii) If $n>1$, then $E_{t}$ is a totally singular $M$-idempotent and $1 \leq \operatorname{red}\left(E_{t}\right)<n$.

Proof. (i) This is obvious.

(ii) In view of Lemma 6.4, it follows from (6.6) that

$$
\lim _{r \rightarrow \infty}\left(A / \rho(A)^{r}\right)=E_{t}
$$

Hence $E_{t}$ is a $Z$-matrix being a limit of $Z$-matrices. But an idempotent matrix has nonnegative eigenvalues and hence $E_{t}$ is an $M$-idempotent.

We now prove the total singularity of $E_{t}$. Note that $F=I-E_{t} \geq 0$ since, by Lemma 6.8 , the diagonal elements of $E_{t}$ are majorized by 1 . Further $E_{t} \neq 1$, since $t>1$ by Lemma 6.4. Thus $F$ is a nonzero nonnegative idempotent. Since $E_{1} E_{t}=0=E_{t} E_{1}$ we also have $E_{1} F=E_{1}=F E_{1}$ and it follows by [1, Theorem 3.14 , p. 41 ], that $F$ is completely reducible and that the maximal eigenvalue of each irreducible block of $F$ is the Perron-Frobenius root of $F$, which is 1 . But this yields the total singularity of $E_{t}$. Finally, since $E_{t} \neq 0$, it follows from Corollary 5.6 that $\operatorname{red}\left(E_{t}\right)<n$. 
THEOREM 6.12. Let $A \in \mathbf{R}^{n n}$, where $n>1$.

(i) Let $A$ be a ZMA-matrix with spectral radius $\rho$. Let $G=E_{t}$ and let $m=$ $\operatorname{red}(G)$. If $\left\{n_{1}, \ldots, n_{m}\right\}$ is the $m$-partition of $n$ which reduces $G$, then there exists a unique normalized positive $U \in \mathcal{U}\left(n_{1}, \ldots, n_{m}\right)$ and a unique $Z P A$-matrix $C \in \mathbf{C}^{m m}$ such that $G=G(U)$ and

$$
A=C \times \times U+\rho G
$$

Further,

$$
\operatorname{spec}(C)=\operatorname{spec}(A) \backslash\{\rho\}
$$

(ii) Conversely, let $m<n$ and let $\left\{n_{1}, \ldots, n_{m}\right\}$ be an $m$-partition of $n$. Let $U$ be a positive element of $\mathcal{U}\left(n_{1}, \ldots, n_{m}\right)$. Let $G=G(U)$ and let $C$ be a ZMA-matrix in $\mathbf{R}^{m m}$ such that $\rho(C)<\rho$. If $A \in \mathbf{R}^{n n}$ satisfies (6.13), then $A$ is a $Z M A$-matrix, $\rho(A)=\rho,\left\{n_{1}, \ldots, n_{m}\right\}$ is the $m$-partition which reduces $G$, and $G=E_{t}$.

REMARK. If $G$ is irreducible $(\operatorname{red}(G)=1)$, then (6.13) and (6.14) become $A=\alpha U+\rho G$, where $U=I-G$ is a positive rank $1, n \times n$ matrix and $-\rho<\alpha<\rho$.

Proof. (i) By Lemma 6.10, $G=E_{t}$ is a totally singular $M$-idempotent and $m=\operatorname{red}(G)<n$. Hence if $\left\{n_{1}, \ldots, n_{m}\right\}$ is the $m$-partition of $n$ which reduces $G$, by Lemma 5.5 there is a unique normalized positive $U \in \mathcal{U}\left(n_{1}, \ldots, n_{m}\right)$ such that $G=G(U)$. Let $B=A-\rho G$. Then $B G=0=G B$ and hence, by Lemma 4.23, $B=C \times \times U$ where $C \in \mathbf{R}^{m m}$ and (6.14) now follows from Corollary 4.22.

We now show that $C$ is a $Z M A$-matrix. Let $i \neq j, 1 \leq i, j \leq m$. Since $G_{i j}=0$ and since by Corollary 2.14 the $Z M A$-matrix $A$ is essentially full, it follows that

$$
0>A_{i j}=B_{i j}=c_{i j} U_{i j}
$$

But $U_{i j}>0$ and hence $c_{i j}<0$. Thus $C$ is an essentially full (hence irreducible) $Z$-matrix.

Finally, for the proof of (i), observe that it follows from Lemma 4.23 and (4.10) that

$$
A^{r}=(C \times \times U)^{r}+\rho^{r} G=\left(C^{r} \times \times U\right)+\rho^{r} G .
$$

The argument of the previous paragraph now shows that $C^{r}$ is an irreducible $Z$ matrix. Hence $C$ is a $Z M A$-matrix.

(ii) Under the conditions of (ii) we again obtain (6.16). Since $\rho(C \times \times U)<$ $\rho \in \operatorname{spec}(A)$ it follows that $G=\lim _{r \rightarrow \infty}(A / \rho)^{r}$. Hence, by $(6.11)$, we deduce that $G=E_{t}$.

To prove that $A$ is a $Z M A$-matrix we note that (6.13) implies that $A_{i j}=c_{i j} U_{i j}$ for $i \neq j$. Since $C$ is an essentially full $Z$-matrix, we have $c_{i j}<0$ and, by assumption, $U_{i j}>0, i \neq j$. Hence $A_{i j}<0, i \neq j$. Next we have

$$
A_{i i}=c_{i i} U_{i i}+\rho\left(I-U_{i i}\right)=\rho I-\left(\rho-c_{i i}\right) U_{i i} .
$$

By Lemma 6.8, $c_{i i} \leq \rho(C)<\rho$, and hence $A$ is an essentially full $Z$-matrix. A similar argument using (6.16) in place of (6.13) shows that all powers of $A$ are essentially full $Z$-matrices. Hence $A$ is a $Z M A$-matrix. 
Theorem 6.18 (Inflation Theorem). Let $A \in \mathbf{R}^{n n}$ and let $\alpha_{p} \in \mathbf{R}$, $p=1, \ldots, t$, satisfy $(6.1)$ and $(6.2)$.

(i) If $A$ is a $Z M A$-matrix with $\operatorname{spec}(A)=\left\{\alpha_{1}, \ldots, \alpha_{t}\right\}$, then there exist uniquely

(a) a sequence of integers $n_{p}, p=1, \ldots, t$, for which

$$
1=n_{1}<\cdots<n_{t}=n,
$$

(b) a sequence of $n_{p-1}$-partitions of $n_{p}, p=2, \ldots, t$,

$$
\left\{m_{1}^{(p)}, \ldots, m_{n_{p-1}}^{(p)}\right\},
$$

(c) a sequence of normalized positive $U_{p} \in \mathcal{U}\left(n_{1}^{p}, \ldots, m_{n_{p-1}}^{p}\right), p=2, \ldots, t$,

(d) a sequence of $Z M A$-matrices $C_{p} \in \mathbf{C}^{n_{p} n_{p}}, p=1, \ldots, t$, such that

$$
\begin{gathered}
C_{1}=\alpha_{1} \in \mathbf{R}^{11}, \\
C_{p}=C_{p-1} \times \times U_{p}+\alpha_{p} G\left(U_{p}\right), \quad p=2, \ldots, t,
\end{gathered}
$$

and

$$
A=C_{t} \text {. }
$$

(ii) Conversely, if (a)-(d) hold, then $A$ is a $Z M A$-matrix and $\operatorname{spec}(A)=\left\{\alpha_{1}, \ldots\right.$, $\left.\alpha_{t}\right\}$.

Proof. (i) The proof is by induction on $t$. If $t=1$, then $n=1$ by Lemma 6.4 and $A=C_{1}$ is given by (6.21) and (6.23), and uniqueness is clear. Let $t>1$ and suppose the result holds for all $Z M A$-matrices with fewer than $t$ pairwise distinct eigenvalues. By Lemma 6.4, $n>1$ and by Theorem 6.12 there is a unique $n_{t-1}<n_{t}=n$, a unique $n_{t-1}$-partition $\left\{m_{1}^{(t)}, \ldots, m_{n_{t-1}}^{(t)}\right\}$ of $n$, and a unique normalized $U \in U\left(m_{1}^{(t)}, \ldots, m_{n_{t-1}}^{(t)}\right)$ such that $A=C_{t-1} \times \times U_{t}+\alpha_{t} G\left(U_{t}\right)$. By Corollary 4.22, $\operatorname{Spec}\left(C_{t-1}\right)=\left\{\alpha_{1}, \ldots, \alpha_{t-1}\right\}$. Hence the inductive assumption applies and the result follows.

(ii) The converse result follows from (ii) of Theorem 6.12 by induction.

EXAMPLE 6.24. Let

$$
A=\left[\begin{array}{rrr}
7 & -3 & -1 \\
-3 & 7 & -1 \\
-1 & -1 & 5
\end{array}\right]
$$

Then $n_{1}=1, \alpha_{1}=3, C_{1}=3$. At the next stage we have $n_{2}=m_{1}^{(2)}=2, \alpha_{2}=6$,

$$
U_{2}=\frac{1}{3}\left[\begin{array}{rr}
2 & \sqrt{2} \\
\sqrt{2} & 1
\end{array}\right] \in U(2), \quad G\left(U_{2}\right)=I-U_{2}=\frac{1}{3}\left[\begin{array}{rr}
1 & -\sqrt{2} \\
-\sqrt{2} & 2
\end{array}\right],
$$

and so

$$
C_{2}=C_{1} \times \times U_{2}+\alpha_{2} G\left(U_{2}\right)=\left[\begin{array}{rr}
4 & -\sqrt{2} \\
-\sqrt{2} & 5
\end{array}\right] .
$$

At the third stage we have $n_{3}=3,\left(m_{1}^{(3)}, m_{2}^{(3)}\right)=(2,1), \alpha_{3}=10$,

$$
U_{3}=\frac{1}{2}\left[\begin{array}{rr|r}
1 & 1 & \sqrt{2} \\
1 & 1 & \sqrt{2} \\
\hline \sqrt{2} & \sqrt{2} & 2
\end{array}\right] \in U(2,1), \quad G\left(U_{3}\right)=\frac{1}{2}\left[\begin{array}{rr|r}
1 & -1 & 0 \\
-1 & 1 & 0 \\
\hline 0 & 0 & 0
\end{array}\right],
$$


and $A=C_{3}=C_{2} \times \times U_{3}+\alpha_{3} G\left(U_{3}\right)$. By Theorem 6.18, $A$ is an $M M A$-matrix. We can continue this process by choosing $n_{4}=5,\left(m_{1}^{(4)}, m_{2}^{(4)}, m_{3}^{(4)}\right)=(2,2,1)$, $\alpha_{4}=20$,

$$
\begin{aligned}
U_{4} & =\left[\begin{array}{ll|ll|r}
.36 & .48 & .36 & .48 & .6 \\
.48 & .64 & .48 & .64 & .8 \\
\hline .36 & .48 & .36 & .48 & .6 \\
.48 & .64 & .48 & .64 & .8 \\
\hline .6 & .8 & .6 & .8 & 1
\end{array}\right] \in \mathcal{U}(2,2,1), \\
G\left(U_{4}\right) & =\left[\begin{array}{lr}
.64 & -.48 \\
-.48 & .64
\end{array}\right] \oplus\left[\begin{array}{rr}
.64 & -.48 \\
-.48 & .64
\end{array}\right] \oplus[0]
\end{aligned}
$$

and obtain the $M M A$-matrix

$$
B=C_{3} \times \times U_{4}+\alpha_{4} G\left(U_{4}\right)=\left[\begin{array}{rrrrr}
15.32 & -6.24 & -1.08 & -1.44 & -.6 \\
-6.24 & 11.68 & -1.44 & -1.92 & -.8 \\
-1.08 & -1.44 & 15.32 & -6.24 & -.6 \\
-1.44 & -1.92 & -6.24 & -11.68 & -.8 \\
-.6 & -.8 & -.6 & -.8 & 5
\end{array}\right] \text {. }
$$

Observe that the inflation operation is associative, i.e. $U_{1} \times \times\left(U_{2} \times \times U_{3}\right)=$ $\left(U_{1} \times \times U_{2}\right) \times \times U_{3}$ for appropriate partitioning.

Corollary 6.25. Let $A \in \mathbf{R}^{n n}$. Then $A$ is a $Z M A$-matrix if and only if its spectral decomposition is $A=\alpha_{1} E_{1}+\cdots+a_{t} E_{t}$, where $\alpha_{1}, \ldots, \alpha_{t}$ satisfy (6.1) and (6.2) and

$$
E_{p}=G\left(U_{p}\right) \times \times U_{p+1} \times \times \cdots \times \times U_{t-1} \times \times U_{t}, \quad p=1, \ldots, t,
$$

where $U_{1}=0 \in \mathbf{R}^{11}$ and $U_{2}, \ldots, U_{t}$ satisfy $(\mathrm{c})$ of Theorem 6.18 .

Proof. Suppose $A$ is a $Z M A$-matrix. Then $\alpha_{1}, \ldots, \alpha_{t}$ satisfy (6.1) and (6.2). The proof of (6.26) is by induction on $t$. If $t=1$, then $n=1$ by Lemma 6.4 and $E_{1}=1=G(0) \in \mathbf{R}^{11}$. Let $t>1$ and suppose that the spectral projections of $C_{t-1}$ in $(6.22)$ are given by

$$
F_{p}=G\left(U_{p}\right) \times \times U_{p+1} \times \times \cdots \times \times U_{t-1}, \quad p=1, \ldots, t-1 .
$$

But then (6.26) follows from Lemma 4.19 and (6.27).

Conversely, suppose that (6.1), (6.2), and (6.26) hold. If $t=1$, then $A=$ $\alpha_{1} \in \mathbf{R}^{11}$ and the result holds. Suppose inductively that the result is true with $t$ replaced by $t-1$. Let $C_{t-1}=\alpha_{1} F_{1}+\cdots+\alpha_{t-1} F_{t-1}$, where the $F_{p}$ are given by $(6.27), p=1, \ldots, t-1$. Then $C_{t-1}$ is a $Z M A$-matrix. If $A$ is given by (6.22) and (6.23), then $A$ has the required spectral decomposition by Lemma 4.19 and is a $Z M A$-matrix by Theorem 6.18 .

Corollary 6.28. Let $A \in \mathbf{R}^{n}$ be a $Z M A$-matrix. Let $A=\alpha_{1} E_{1}+\cdots+\alpha_{t} E_{t}$ be its spectral decomposition. Let $\beta_{p} \in \mathbf{R}, p=1, \ldots, t$, satisfy $\beta_{1}<\beta_{2}<\cdots<\beta_{t}$ and suppose that $B=\beta_{1} E_{1}+\cdots+\beta_{t} E_{t}$. Then

(i) $B$ is a $Z M A$-matrix if and only if $-\beta_{2}<\beta_{1}$.

(ii) $B$ is a ZMO-matrix if and only if $-\beta_{2}=\beta_{1}$.

(iii) $B$ is an $M M A$-matrix if and only if $0 \leq \beta_{1}$. 
ProOF. (i) By Corollary 6.25 the projections $E_{p}, p=1, \ldots, t$, satisfy (6.26). Then the result follows from Corollary 6.25 .

(ii) Follows from (i) and Theorem 3.7.

(iii) Follows from (i) and Theorem 3.9.

EXAMPLE 6.29. Let $A$ be the matrix of Example 6.24. We then have $\alpha_{1}=3$, $\alpha_{2}=6, \alpha_{3}=10$. Further, in the notation of Example 6.24,

$$
\begin{gathered}
E_{1}=1 \times \times U_{2} \times \times U_{3}=\frac{1}{3}\left[\begin{array}{lll}
1 & 1 & 1 \\
1 & 1 & 1 \\
1 & 1 & 1
\end{array}\right], \\
E_{2}=G\left(U_{2}\right) \times \times U_{3}=\frac{1}{6}\left[\begin{array}{rrr}
1 & 1 & -2 \\
1 & 1 & -1 \\
-2 & -2 & 4
\end{array}\right], \\
E_{3}=G\left(U_{3}\right)=\frac{1}{2}\left[\begin{array}{rrr}
1 & -1 & 0 \\
-1 & 1 & 0 \\
0 & 0 & 0
\end{array}\right]
\end{gathered}
$$

If $\beta_{1}=3, \beta_{3}=3, \beta_{3}=5$, then

Then $B$ is a $Z M O$-matrix and

$$
B=\left[\begin{array}{rrr}
2 & -3 & -2 \\
-3 & 2 & -2 \\
-2 & -2 & 1
\end{array}\right]
$$

$$
B^{2}=\left[\begin{array}{rrr}
17 & -8 & 0 \\
-8 & 17 & 0 \\
0 & 0 & 9
\end{array}\right]
$$

which is a completely reducible $M$-matrix.

We now strengthen Lemma 6.8 for $Z M E$-matrices.

COROLlaRY 6.30. Let $A \in \mathbf{R}^{n n}$ be a $Z M E$-matrix. If $n>1$, then

$$
\left|a_{k k}\right|<\rho(A), \quad k=1, \ldots, n .
$$

PROOF. In view of Lemma 3.10 and the result that for a $Z M E$-matrix $A$ we have $\rho(A) \in \operatorname{spec}(A)$ it is enough to prove the lemma for a $Z M A$-matrix $A$. Since $n>1$, we may write $A$ in the form (6.13) where $\rho=\rho(A)$ and $C$ is an $m \times m Z M A$-matrix where $m<n$ and $\rho(C)<\rho$. By Lemma 6.8 we have $\left|c_{k k}\right| \leq \rho(C), k=1, \ldots, m$. The result now follows from (6.17) and the observation that the diagonal elements of $U$ are positive.

\section{Roots of $Z M E$-matrices and eventual $Z$-matrices.}

THEOREM 7.1. Let $A \in \mathbf{R}^{n n}$ and let $r$ be a positive integer.

(i) If $A$ is a $Z M A$-matrix and $r$ is odd, then there is a unique $Z M A$-matrix $B$ which satisfies $B^{r}=A$.

(ii) If $A$ is a $Z M O$-matrix and $r$ is odd, then there is a unique $Z M O$-matrix $B$ which satisfies $B^{r}=A$.

(iii) If $A$ is an MMA-matrix, then there is a unique $M M A$-matrix $B$ which satisfies $B^{r}=A$. 
Proof. (i) Since $A$ is a $Z M A$-matrix we may write it in the form

$$
A=\alpha_{1} E_{1}+\cdots+\alpha_{t} E_{t}
$$

by Corollary 6.25 , where $\alpha_{1}, \ldots, \alpha_{t}$ satisfy (6.1) and (6.2). Let $\beta_{p}$ be the real $r$ th root of $\alpha_{p}, p=1, \ldots, t$, and define $B$ by

$$
B=\beta_{1} E_{1}+\cdots+\beta_{t} E_{t} .
$$

Then $A$ is a $Z M A$-matrix by Corollary 6.28 .

To prove uniqueness, let $C$ be a $Z M A$-matrix such that $C^{r}=A$. Then, by (6.5) and the simplicity of the eigenvalue $\alpha_{1}$ of $A$, the spectral decomposition of $C$ is given by

$$
C=\gamma_{1} F_{1}+\cdots+\gamma_{t^{\prime}} F_{t^{\prime}}
$$

where $\gamma_{1}, \ldots, \gamma_{t^{\prime}}$ satisfy (6.1) and (6.2). But then it follows from $C^{r}=A$ that $t=t^{\prime}, \gamma_{p}=\beta_{p}$, and $F_{p}=E_{p}, p=1, \ldots, t$.

(ii) The proof is similar.

(iii) The proof is similar; let $\beta_{p}$ be the nonnegative $r$ th root of $\alpha_{p}, p=1, \ldots, t$.

For our next theorem we require a somewhat more precise form of Theorem 7.1.

LemMA 7.4. Let $A, B \in \mathbf{R}^{n n}$ and let $r$ be a positive integer. If $A$ is a $Z M A$ matrix (ZMO-matrix), $B^{r}=A$, and the spectrum of $B$ satisfies (6.1) and (3.2), then $B$ is a $Z M A$-matrix (ZMO-matrix).

Proof. (i) Since $A$ is a $Z M E$-matrix we may write it in the form

$$
A=\alpha_{1} E_{1}+\cdots+\alpha_{t} E_{t}
$$

by Corollary 6.25, where $\alpha_{1}, \ldots, \alpha_{t}$ satisfy $(6.1)$ and $\left|\alpha_{1}\right| \leq \alpha_{2}$. Since $\operatorname{spec}(B)=$ $\left\{\beta_{1}, \ldots, \beta_{t^{\prime}}\right\}$ also satisfies (6.1) it follows from $B^{r}=A$ that $t=t^{\prime}$ and $\beta_{p}^{r}=\alpha_{p}$, $p=1, \ldots, t$. Since $\alpha_{1}$ is a simple eigenvalue of $A$, the eigenvalue $\beta_{1}$ of $B$ is also simple. Hence, since $\beta_{p} \neq 0, p=2, \ldots, t$, it follows as in the proof of Theorem 7.1 that the spectral decomposition of $B$ is given by

$$
B=\beta_{1} E_{1}+\cdots+\beta_{t} E_{t} .
$$

Thus if $A$ is a $Z M A$-matrix ( $Z M O$-matrix) the matrix $B$ is also a $Z M A$-matrix (ZMO-matrix) by Corollary 6.28 .

THEOREM 7.7. Let $A \in \mathbf{R}^{n n}$ be an eventual $Z$-matrix.

(i) If some power of $A$ is an irreducible $Z$-matrix, then $A$ is a $Z M E$-matrix.

(ii) If some even power of $A$ is an irreducible $Z$-matrix, then $A$ is a $Z M A$-matrix.

(iii) If there is a power of $A$ which is an irreducible $Z$-matrix and an odd power of $A$ which is an $M$-matrix, then $A$ is an MMA-matrix.

Proof. (i) By Lemma 3.1, $\operatorname{Spec}(A)=\left\{\alpha_{1}, \ldots, \alpha_{t}\right\}$ is real and satisfies (6.1), where $\alpha_{1}$ is a simple eigenvalue and the associated projection $E_{1}$ is positive. If $r$ is a sufficiently large odd integer, then $A^{r}$ is a $Z M$-matrix and $A^{r}$ is irreducible since $\alpha_{1}^{r}$ is a simple eigenvalue of $A^{r}$ and its associated projection $E_{1}$ is positive. Hence $A^{r}$ is a $Z M E$-matrix by Corollary 3.8. But since the spectrum of $A$ satisfies (6.1) it follows from Lemma 7.4 that $A$ is also a $Z M E$-matrix.

(ii) This follows immediately from (i) and Theorem 3.6, since the irreducibility of an even power of $A$ implies the irreducibility of $A^{2}$.

(iii) This follows from (i) and Theorem 3.9, since the irreducibility of a power of $A$ implies the irreducibility of $A$. 
8. Exponentials and logarithms. In this section we allow matrices to be complex in some cases. This small increase in generality would also be possible in some other sections.

Let $A \in \mathbf{C}^{n n}$. Then $B=e^{A}$ may be defined by means of the usual power series. If the spectral decomposition of $A$ is given by

$$
A=\alpha_{1} E_{1}+\cdots+\alpha_{t} E_{t},
$$

then the spectral decomposition of $B$ is

$$
B=\beta_{1} E_{1}+\cdots+\beta_{t} E_{t},
$$

where

$$
\beta_{p}=e^{\alpha_{p}}, \quad p=1, \ldots, t .
$$

Evidently if $\alpha_{p}$ is real and

$$
\alpha_{1}<\alpha_{2} \cdots<\alpha_{t}
$$

(which is the same as (6.1)), then

$$
0<\beta_{1}<\cdots<\beta_{t} .
$$

It is also possible to define a logarithm of an arbitrary nonsingular matrix in $\mathbf{C}^{n n}$ (see [4]), but here we shall only consider the logarithm of a matrix $B$ whose spectral decomposition satisfies (8.2) and (8.5). In that case, we may define $A=\log B$ as the matrix given by $(8.1)$ where

$$
\alpha_{p}=\log \beta_{p}, \quad p=1, \ldots, t .
$$

We then have the usual inverse function relations: $e^{\log B}=B$ and $\log e^{A}=A$.

Matrices which are essentially $M M A$-matrices were defined in Definition 1.1(vi). Evidently if $A$ is essentially an $M M A$-matrix, we may choose a nonnegative $s$ so that $A+s I$ is an $M M A$-matrix. Observe that a $Z M E$-matrix is essentially an $M M A$-matrix.

THEOREM 8.7. (i) Let $A \in \mathbf{R}^{n n}$ be essentially an MMA-matrix. Then $e^{A}$ is a nonsingular $M M A$-matrix.

(ii) Let $B \in \mathbf{R}^{n n}$ be a nonsingular MMA-matrix. Then $\log B$ is essentially an MMA-matrix A.

(iii) Let $A \in \mathbf{C}^{n n}$ and suppose that $e^{A}$ is an MMA-matrix. Then $A$ is essentially an $M M A$-matrix if and only if $\operatorname{spec}(A)$ is real.

Proof. (i) Since $A+s I$ is an $M M A$-matrix for some $s \in \mathbf{R}$, it follows easily from Corollary 6.25 that the matrix $A$ has a spectral decomposition given by (8.1) and (8.4). Then $B$ satisfies (8.2), (8.5) and so by Corollary $6.28(i i i), B$ is a nonsingular $M M A$-matrix.

(ii) The spectral decomposition of $B$ satisfies (8.2) and (8.5). Hence the spectral decomposition of $A=\log B$ satisfies (8.1) and (8.4). By Corollary 6.28, $A$ is essentially an $M M A$-matrix.

(iii) If $A$ is essentially an $M M A$-matrix, then its spectrum is real. Conversely, suppose that $\operatorname{spec}(A)$ is real and that $B=e^{A}$ is an $M M A$-matrix with spectral decomposition satisfying (8.2) and (8.5). Then $\alpha_{p}=\log \beta_{p}, p=1, \ldots, t$, since $e^{t}$ is 
a strictly monotonic function on $\mathbf{R}$ and (8.1) and (8.4) hold. Hence $A=\log B$ and so $A$ is essentially an $M M A$-matrix by (ii).

If $B$ is a nonsingular $M M A$-matrix it is easy to see that $\log A$ is an $M M A$-matrix if and only if $\beta_{1} \geq 1$ in (8.5) and a $Z M E$-matrix if and only if $\left(\beta_{2}\right)^{-1} \leq \beta_{1}$.

DEFINITIONS 8.8. Let $A \in \mathbf{C}^{n n}$.

(i) The semigroup $\left\{e^{s A}: s \geq 0\right\}$ will be called the semigroup with infinitesimal generator $A$ and will be denoted by $S(A)$.

(ii) The semigroup $S(A)$ will be called a real (nonnegative, $M M A-$, etc.) semigroup if $e^{s A}$ is a real (nonnegative, $M M A-$, etc.) matrix whenever $s>0$.

It is well known that the semigroup $S(A)$ is nonnegative (positive) if and only if $A$ is essentially nonnegative (and irreducible), i.e. $A+s I$ is nonnegative (and irreducible) for some $s \in \mathbf{R}$ [9, pp. 257 and 260]. We prove an analogous result.

THEOREM 8.9. Let $A \in \mathbf{C}^{n n}$. Then the following are equivalent.

(i) $e^{s A}$ is an eventual $Z$ matrix for all sufficiently small positive $s$ and $S(A)$ contains an irreducible $Z$-matrix.

(ii) $A \in \mathbf{R}^{n n}$, $\operatorname{spec}(A) \subseteq \mathbf{R}$, and $S(A)$ contains an eventual $Z$-matrix $C$ some power of which is an irreducible $Z$-matrix.

(iii) $A$ is essentially an $M M A$-matrix.

(iv) $S(A)$ is an $M M A$-semigroup.

ProOF. (i) $\Rightarrow$ (ii). As is easily proved

$$
A=\lim _{s \rightarrow 0+}\left(e^{s A}-I\right) / s .
$$

As an eventual $Z$-matrix is real (by definition) it follows from (8.10) that $A$ is real. Let $e^{s^{\prime} A}$ be an irreducible $Z$-matrix. Clearly $s^{\prime}>0$. Let

$$
B_{k}=e^{\left(s^{\prime} / k\right) A}, \quad k=1,2, \ldots
$$

Then, for sufficiently large $k, B_{k}$ is an eventual $Z$-matrix some power of which is an irreducible $Z$-matrix since $B_{k}^{k}=B_{1}$. By Theorem 7.7, $B_{k}$ is an $M M A$-matrix and hence $\operatorname{spec}\left(B_{k}\right)$ is real for sufficiently large $k$. Hence, by (8.10),

$$
\lim _{k \rightarrow \infty} k\left(B_{k}-I\right) / s^{\prime}=A,
$$

and it follows that $\operatorname{spec}(A)$ is real.

(ii) $\Rightarrow$ (iii). By Theorem 7.7 the matrix $C$ is an $M M A$-matrix. Since $e^{s A}=C$ for some positive $s$, it follows by Theorem 8.7(iii) that $s A$ is an $M M A$-matrix. Thus (iii) follows.

(iii) $\Rightarrow$ (iv). By Theorem $8.7(\mathrm{i})$.

(iv) $\Rightarrow$ (i) is trivial.

9. Positive diagonal similarity to symmetric matrices. We shall show that for a symmetric $Z M A$-matrix the matrices which occur in the inflation construction of Theorem 6.18 are again symmetric.

LEMma 9.1. Let $A$ be a $Z M A$-matrix and let $U$ and $C$ be the matrices which satisfy (6.13) in Theorem 6.12. Then $U$ and $C$ are symmetric if and only if $A$ is symmetric.

PROOF. If $U$ and $C$ are symmetric, the symmetry of $A$ follows immediately from (6.13). Conversely, let $A$ be symmetric. Then $G$ is symmetric since, by assumption, 
$G$ is the projection associated with $\rho(A)$. But then each irreducible component $G_{i i}$, $i=1, \ldots, m$, of $G$ is symmetric and it now follows from the normalization (4.15) that $u_{i}=v_{i}, i=1, \ldots, m$. Hence $U$ is symmetric.

THEOREM 9.2. Let $A$ be a $Z M A$-matrix with $t$ pairwise distinct eigenvalues. Then $A$ is symmetric if and only if the matrices $C_{1}, \ldots, C_{t-1}, U_{2}, \ldots, U_{t}$ which occur in Theorem 6.18 are symmetric.

ProOF. If $C_{1}, \ldots, C_{t-1}, U_{2}, \ldots, U_{t}$ are symmetric, it follows immediately from (6.22) that $A$ is symmetric.

Conversely suppose that $A$ is symmetric. Our proof is by induction on $t$. If $t=1$, there is nothing to prove. Let $t>1$. By Lemma 8.1 it follows from (6.22) and (6.23) that $C_{t-1}$ and $U_{t}$ are symmetric. Since $C_{t-1}$ has $t-1$ pairwise distinct eigenvalues, by Corollary 4.22 , it follows by our inductive assumption that $C_{1}, \ldots, C_{t-2}, U_{2}, \ldots, U_{t-1}$ are symmetric. This yields the result.

DEFINITION 9.3. (i) A diagonal matrix with positive diagonal elements will be called a positive diagonal matrix.

(ii) Let $A, B \in \mathbf{C}^{n n}$ (or $\mathbf{R}^{n n}$ ). Then $A$ is called positively diagonally similar to $B(A \sim B)$ if there is an $n \times n$ positive diagonal matrix $X$ for which $B=X^{-1} A X$.

We shall show that an irreducible $Z M A$-matrix is positively diagonally similar to a unique symmetric $Z M A$-matrix. We begin with two lemmas. They are essentially special cases of a theorem dating from Parter and Youngs [8], see also Engel and Schneider [2]. But as the proofs in the needed cases are very simple they are given here for the sake of completeness.

LEMMA 9.4. Let $A$ and $A^{\prime}$ be two essentially full symmetric matrices in $\mathbf{R}^{n n}$ and suppose that $A \sim A^{\prime}$. Then $A=A^{\prime}$.

ProOF. Let $A^{\prime}=X^{-1} A X$, where $X$ is a positive diagonal matrix. Let $1 \leq$ $h, k \leq n, h \neq k$. Then $a_{h k}=a_{k h} \neq 0$ and $x_{h}^{-1} a_{h k} x_{k}=x_{k}^{-1} a_{k h} x_{h}$. It follows that $x_{h}=x_{k}$ and hence $X$ is a positive multiple of the identity. The lemma follows.

LEMMA 9.5. Let $\left\{n_{1}, \ldots, n_{m}\right\}$ be an m-partition of $n$ and $U \in \mathcal{U}\left(n_{1}, \ldots, n_{m}\right)$ be positive. Then there is a unique symmetric $U^{\prime} \in \mathcal{U}\left(n_{1}, \ldots, n_{m}\right)$ such that $U^{\prime} \sim U$. Further, $U^{\prime}$ is positive.

PROOF. We may assume that $u_{h k}=u_{h} v_{k}, h, k=1, \ldots, n$. (Here the subscripts refer to elements.) We let $Y$ be the diagonal matrix for which $y_{k k}=\left(v_{k} / u_{k}\right)^{1 / 2}$, $k=1, \ldots, n$. Then $U^{\prime}=Y^{-1} U Y$ is a symmetric matrix. Since $\operatorname{rank}\left(U^{\prime}\right)=1$ and $\operatorname{trace}\left(U_{11}^{\prime}\right)=1, i=1, \ldots, m$, it follows that $U^{\prime} \in \mathcal{U}\left(n_{1}, \ldots, n_{m}\right)$. Evidently $U^{\prime}$ is positive.

The uniqueness of $U^{\prime}$ follows from the Lemma 9.4.

THEOREM 9.6. Let $A \in \mathbf{R}^{n n}$ be a $Z M A$-matrix. Then there exists a unique symmetric $Z M A$-matrix $A^{\prime}$ which is positively diagonally similar to $A$.

PROOF. The proof of the existence of $A^{\prime}$ is by induction on $t$, the number of distinct eigenvalues of $A$. If $t=1$, then $n=1$ and the result is true. Let $n>1$ and suppose the result holds for all irreducible $Z M A$-matrices $C$ with fewer than $t$ distinct eigenvalues. Let $U \in \mathcal{U}\left(m_{1}, \ldots, m_{t-1}\right) \subseteq \mathbf{R}^{n n}$ and $C \in \mathbf{R}^{m m}$ be matrices satisfying (6.13). By inductive assumption there exists a positive diagonal matrix $X \in \mathbf{R}^{m m}$ such that $C^{\prime}=X^{-1} C X$. By Lemma 8.5, there is a positive 
diagonal matrix $Y \in \mathbf{R}^{n n}$ such that $U^{\prime}=Y^{-1} U Y$ is a symmetric positive matrix in $\mathcal{U}\left(m_{1}, \ldots, m_{t-1}\right)$. Let $Z \in \mathbf{R}\left(m_{1}, \ldots, m_{t-1}\right)$ be the diagonal matrix given by $Z_{i}=x_{i} Y_{i} \in \mathbf{R}^{m_{i} m_{i}}, i=1, \ldots, m$. Then $C^{\prime} \times \times U^{\prime}=Z^{-1}(C \times \times U) Z$. Also $G\left(U^{\prime}\right)$ is symmetric and $G\left(U^{\prime}\right)=Z^{-1} G(U) Z$. Hence, if $\rho=\rho(A)$,

$$
A^{\prime}=C \times \times U+\rho G(U)=Z^{-1}\left(C \times \times U+\rho G(U) Z=Z^{-1} A Z\right.
$$

is a symmetric martrix. Since $A^{\prime}$ is positively diagonally similar to $A$ it follows that $A$ is a $Z M A$-matrix.

Since, by Corollary 2.14, $A$ is essentially full, the uniqueness of $A$ follows from Lemma 9.4 .

A different proof and a generalization of Theorem 9.6 will appear in Hershkowitz and Schneider [6]. In view of Lemma 3.10 similar results hold on the positive diagonal similarity of $Z M O$-matrices to symmetric $Z M O$-matrices.

\section{REFERENCES}

1. A. Berman and R. J. Plemmons, Nonnegative matrices in the mathematical sciences, Academic Press, New York, 1979.

2. G. M. Engel and H. Schneider, Cyclic and diagonal products on a matrix, Linear Algebra Appl. 7 (1973), 301-355.

3. M. Fiedler and V. Ptak, On matrices with non-positive off-diagonal elements and positive principal minors, Czechoslovak Math. J. 12(87) (1962), 382-400.

4. M. Fiedler and H. Schneider, Analytic functions of $M$-matrices and generalizations, Linear and Multilinear Algebra 13 (1983), 185-201.

5. F. R. Gantmacher, The theory of matrices, Chelsea, New York, 1959.

6. D. Hershkowitz and H. Schneider, Matrices with a sequence of accretive powers, Israel J. Math. (to appear).

7. A. M. Ostrowski, Über die Determinanten mit überwiegender Hauptdiagonale, Comment. Math. Helv. 10 (1937), 69-96.

8. S. V. Parter and J. W. T. Youngs, The symmetrization of matrices by diagonal matrices, J. Math. Anal. Appl. 4 (1962), 102-110.

9. R. S. Varga, Matrix iterative analysis, Prentice-Hall, Englewood Cliffs, N.J., 1962.

Institute of Mathematics, Hebrew University, Jerusalem, ISRAel

Department of Mathematics, Cornell University, IthaCA, NeW York 14853 (Current address of Shmuel Friedland)

Department of MATHEMAtics, Statistics AND Computer SCIENCE, UNiversity OF Illinois, CHICAGO, IlLiNOIS 60680

DEPARTMENT OF MATHEMATICS, UNIVERSITY OF WISCONSIN-MADISON, MADISON, WISCONSIN 53706 (Current address of Hans Schneider)

DEPARTMENT OF MATHEMATICS, TECHNION-ISRAEL INSTITUTE OF TECHNOLOGY, HAIFA 32000, ISRAEL (Current address of Daniel Hershkowitz) 